Martínez-Garrido, Cynthia \& Murillo, F. Javier (2016). Incidencia de la distribución del tiempo no lectivo de los docentes en Educación Primaria en el aprendizaje de sus estudiantes. RELIEVE, 22(2), art. 1. doi: http://dx.doi.org/10.7203/relieve.22.2.9433 Revista ELectrónica de Investigación
y EValuación Educativa

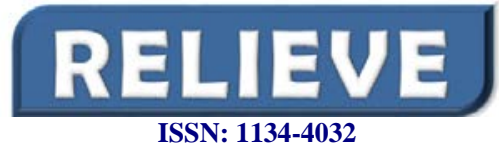

ISSN: 1134-4032
e-Journal of Educational Research,

Assessment and Evaluation

\title{
Incidencia de la distribución del tiempo no lectivo de los docentes en Educación Primaria en el aprendizaje de sus estudiantes
}

\author{
Impact of the Distribution of Non-Instructional Time of Primary-School Teachers on Student \\ Learning
}

\author{
Martínez-Garrido, Cynthia (1) \& Murillo, F. Javier ${ }^{(2)}$
}

(1) Universidad de Zaragoza (2) Universidad Autónoma de Madrid

\begin{abstract}
Resumen
Esta investigación busca determinar la incidencia del porcentaje de tiempo que los docentes iberoamericanos de Educación Primaria destinan a las diferentes tareas no lectivas sobre el logro académico de sus estudiantes en Matemáticas y en Lectura. Para ello se ha realizado un estudio con 256 docentes de 9 países de Iberoamérica y sus 5.610 estudiantes. Concretamente se ha recogido información de la distribución del tiempo de los docentes, así como de las características de los Fecha de estudiantes y su entorno, y de su rendimiento en Matemáticas y en Lectura, mediante pruebas recepción previamente validadas para todos los países, al principio y al final del curso. Utilizando Modelos 12 Abril 2015 Multinivel de cuatro niveles de análisis (estudiante, aula, escuela y país), se ha encontrado que una mayor dedicación a tres tareas mejora significativamente el logro de los estudiantes: la preparación de las clases, la corrección de evaluaciones y el trabajo con familias. Y, por el contrario, una mayor dedicación a tareas administrativas hace que disminuya el logro de los estudiantes. Con estos resultados se aportan evidencias empíricas acerca de la necesidad de que los docentes tengan unas condiciones laborales adecuadas para desempeñar adecuadamente su trabajo. Así, es necesario considerar que el trabajo de los docentes no es solo estar frente al grupo, también hay que dejarles tiempo y espacios apropiados para que prepararen su trabajo en el aula. Igualmente, todo lo que sea minimizar las tareas administrativas a realizar por los docentes redundará en un mayor aprendizaje de los estudiantes, seguramente porque los docentes se dedicarán "a lo importante".
\end{abstract}

Palabras clave: Enseñanza Eficaz, Tiempo de los docentes, Rendimiento Académico, Análisis Multinivel, Iberoamérica

\begin{abstract}
This research aims to determine the incidence of the percentage of time that Latin American teachers of Primary Education allocated to different no teaching related tasks on the academic achievement of students in Mathematics and Language. We have conducted a study with 256 teachers from 9 countries in Latin America and 5,610 students. The information was collected from the teachers' distribution time as well as the characteristics of students and their environment, and performance in Mathematics and Language through validated tests for all countries at the beginning and at the end the course. Using Multilevel Models with four levels of analysis (student, classroom, school and country), it has been found that an increased focus on three tasks significantly improves student achievement: the lesson preparation, correction of assessments, and work with families. And, conversely, a greater emphasis on administrative tasks lowers achieving students. With these results, empirical evidence is provided on the need for teachers to have suitable working conditions to adequately perform their jobs. Thus, it is necessary to consider that the work of teachers is not only being with the group, you also have to let time and prepare appropriate spaces for their work in the classroom. Similarly, whatever is minimizing administrative tasks performed by teachers result in greater student learning, probably because teachers are devoted "to the important"
\end{abstract}

Fecha de aprobación 20 Julio 2016

Fecha de publicación 20 Julio 2016

Keywords: Teaching effectiveness, Teacher' time, Academic Performance, Multilevel Analysis, Iberoamerica.

F. Javier Murillo. Facultad de Formación de Profesorado y Educación. Universidad Autónoma de Madrid. 
Los docentes en todo el mundo se encuentran sobresaturados de trabajo y sometidos a fuertes presiones de todo tipo, tanto intra como extraescolares. Efectivamente, los profesores tanto de Educación Primaria como de Secundaria, además de sus tareas docentes frente al grupo, deben planificar clases, preparar y corregir exámenes y ejercicios, atender a los estudiantes fuera del aula, reunirse con familias, seguir aprendiendo, colaborar con el resto de docentes, asistir a las actividades extraescolares, realizar tareas administrativas... Con ello, no es de extrañar que el colectivo docente sufra importantes casos de estrés y burnout laboral (Kyriacou, 1987; Vandenberghe \& Huberman, 1999). Sin embargo, parece claro que no todas las tareas son igualmente relevantes, no incidirá lo mismo en el aprendizaje de sus estudiantes el tiempo que dedica a preparar las clases que a completar la burocracia que se le exige.

Si partimos de la base que los docentes y su trabajo son la clave del sistema educativo, y de que la mejora de su desempeño es uno de los fundamentos para la optimización del desarrollo de los estudiantes, parece necesario considerar que la mejora de las condiciones laborales de los docentes, fomentando la realización de las actividades mas útiles y minimizando el tiempo dedicado a hacer otras que tan sólo parecen tener incidencia. Incluso puede ser que algunas tareas influyan negativamente en el aprendizaje de los estudiantes, por lo que supone de restar tiempo a otras mas importantes, lo que llevaría a la necesidad incluso de eliminarlas.

En coherencia con ello, existe una fructífera línea de investigación que busca determinar cómo distribuyen su tiempo los docentes $\mathrm{y}$, especialmente, qué tareas tienen una mayor incidencia sobre el aprendizaje que otras (p.e. Husman, Duggan \& Fishman, 2014; Lavy, 2011; Pennings et al., 2014; Shidler, 2009; Vannest, Hagan-Burke \& Parker, 2006).

En América Latina, donde uno de cada tres profesores combina su actividad docente con otra actividad laboral (Murillo y Román,
2012), el interés para mejorar el desempeño de los docentes se ha centrado más en potenciar evaluaciones centradas en los conocimientos del profesor -en frecuencia con repercusiones negativas- que en mejorar sus condiciones de trabajo (aun sabiendo que estas últimas son fundamentales para que el docente haga un buen trabajo). Tal vez por ello, se sabe poco sobre a qué tareas se dedican los docentes latinoamericanos y cómo las distribuyen; y menos información se tiene sobre cómo esas actividades indicen en el aprendizaje de sus estudiantes.

Con esta investigación se pretende conocer qué tareas no-lectivas de las que desarrolla el docente tienen un mayor impacto en el desarrollo de sus estudiantes y estimar su aportación. Para alcanzar ese propósito, se lleva a cabo una estudio ex post facto con datos de 256 docentes de nueve países de Iberoamérica. Los datos recogidos son analizados a través de la estrategia metodológica multinivel siguiendo un planteamiento de valor agregado, esto es, medimos la mejora del desempeño de los estudiantes descontando la aportación de otras variables como el rendimiento previo, la situación socioeconómica y cultural de las familias, el nivel socioeconómico del barrio donde se ubica la escuela, el género de los estudiantes, su origen y lengua materna. El control de estas variables permite determinar la influencia real de las variables explicativas (relativas al porcentaje de tiempo que los docentes destinan a una u otra actividad). Una vez presentados y discutidos los resultados, se ofrecen algunas claves y recomendaciones para la mejora de las políticas educativas.

Tradicionalmente se suele organizar la distribución del tiempo de los docentes en cuatro tipos de actividades: a) docencia directa en el aula, b) preparación y planificación de las clases, c) trabajo en equipo con su compañeros, y d) otras, entre las que se incluyen: reuniones con padres, tutorías con estudiantes, y tareas administrativas. Esta distribución es muy diferente de un país a otro. Así, con datos de Europa (Eurydice, 2013), las 
horas lectivas de los docentes no universitarios varían entre 12 y 36 horas por semana de un país a otro. Como pauta general puede decirse que el número de horas lectivas disminuye con el nivel en el que se enseña, desde Educación Infantil, hasta Bachillerato. Así, en la Unión Europea el promedio de horas de docencia directa es del 60,2\% en Infantil; 47,9\% en Primaria; $41,6 \%$ en Secundaria Inferior y $40,1 \%$ en Secundaria Superior. También en América Latina hay grandes diferencias por países. En un estudio de hace ya más de una década (Gajardo, 2002) se encontró que en México el tiempo dedicado a la instrucción no supera el $30 \%$ del total, mientras que para Chile y Ecuador, el tiempo de docencia fluctúa entre el $51 \%$ y $67 \%$ del total del tiempo de clase.

Más interesantes son los trabajos que relacionan la distribución del tiempo de los docentes con el aprendizaje de los estudiantes. Uno de los más influyentes es el desarrollado a principios de la década de los 80 por S. Gibson (1984). En él se estudió cómo distribuían su tiempo los profesores que conseguían que sus estudiantes aprendieran más y menos de lo previsto. Es decir, cuáles son las diferencias de cantidad de tiempo que destinan los profesores de alta y baja eficacia a las diferentes tareas. De entre sus resultados encontrados, dos son especialmente interesantes para este trabajo. Por una parte, los docentes más eficaces dedican menos tiempo a actividades lectivas que los menos eficaces (234 minutos versus 271 minutos, respectivamente); por otra, que los docentes que consiguen que sus alumnos aprendan dedican el doble de tiempo a planificar las clases frente a los que consiguen peores resultados de sus alumnos (39,5 minutos diarios frente a los 16,3). Este resultado lleva a pensar que la actividad del docente que más está asociada con el aprendizaje de los estudiantes es el la planificación de las clases.

Ese resultado es coherente con numerosos estudios que han encontrado que el elemento clave dentro del estudio del tiempo de los docentes no es tanto cómo se distribuye sino qué cantidad de él dedican a la preparación de las clases (Hall \& Harding, 2003; Hiebert, Morris \& Spitzer, 2009; Husman, Duggan \& Fishman, 2014; Martínez-Garrido, 2015; Pennings et al., 2014; Shidler, 2009; Vannest, Hagan-Burke \& Parker, 2006). Estos trabajos, en su gran parte, provienen de análisis de las escuelas anglosajonas y europeas, siendo escasos los datos disponibles de los docentes en Iberoamérica (Albornoz, 1996; Gajardo, 2002). El estudio elaborado por Gran, Hindman y Stronge (2010), por ejemplo, apunta a que el 52\% del éxito (o fracaso) logrado por los estudiantes en Matemáticas depende del tiempo con que el docente preparó la asignatura; y en el trabajo de Nye, Konstantopoulos y Hedges (2004) se muestra que la eficacia que demuestren los docentes en la planificación y organización de las sesiones aumentará 0,5 desviaciones típicas el rendimiento de los estudiantes a lo largo del curso.

Como señalábamos, en el ámbito iberoamericano pocas investigaciones se han centrado en analizar la influencia que la distribución del tiempo de los docentes puede provocar en el logro académico de los estudiantes (Gajardo, 2002). Destaca, sin embargo, el trabajo desarrollado en Chile por Carlos Concha (1996), donde se ha encontrado que la diferencia entre las escuelas exitosas y las no exitosas se debe, en gran medida, a la disponibilidad de tiempo que el docente tiene para planificar la asignatura y gestionar los avances que los estudiantes van logrando a lo largo del curso, así como para dar clase a sus estudiantes. La capacidad de innovación y mejora de las escuelas, según el trabajo de Montecinos (2003), depende directamente del hecho de que los docentes cuenten con tiempo directamente focalizado a un currículum riguroso y mejores formas de atender a la diversidad de sus estudiantes. Según los datos del informe TALIS "Estudio internacional sobre enseñanza-aprendizaje 2013" de la OCDE (2013) se estima que el $47 \%$ de los profesores mexicanos y el $25 \%$ de los docentes chilenos señalan que contar con tiempo para el desarrollo profesional es una prioridad de los 
docentes. De media, el 22\% de los docentes de América Latina encuestados apunta a que es una necesidad. También el trabajo del LLECE (2010), donde se encontró que el rendimiento académico de los estudiantes puede disminuir un $3 \%$ si sus docentes no cuentan con tiempo suficiente para preparar sus clases y evaluar los trabajos de los estudiantes. Los resultados de la Investigación Iberoamericana se Eficacia Escolar también apuntan a que el tiempo que el docente dedica a la preparación de las clases es una variables que índice de forma significativa en el desarrollo de los estudiantes (Murillo, 2007). Murillo y Hernández-Castilla (2012), por último, encontraron relación entre el tiempo de planificación de los docentes y la satisfacción de los alumnos con la escuela.

Partiendo de estos antecedentes, esta investigación busca "determinar la incidencia del porcentaje de tiempo que los docentes iberoamericanos de Educación Primaria destinan a las diferentes tareas no lectivas sobre el logro académico de sus estudiantes en Matemáticas y en Lectura”.

\section{Método}

Para alcanzar dicho objetivo se lleva a cabo un estudio ex post-facto mediante el enfoque metodológico de los Modelos Multinivel con cuatro niveles de análisis -estudiante, aula, escuela y país-, y con un planteamiento de valor agregado, esto es, controlando las variables de producto mediante variables de ajuste tales como el rendimiento previo de lo estudiantes, la situación socio-económica y cultural de sus familias y, en este caso, las características de sus docentes para ver la influencia real de las variables explicativas (Miñano y Castejón, 2011).

\section{Variables}

Esta investigación sigue un planteamiento de Valor añadido, es decir, se entiende por logro escolar el desempeño de los estudiantes controlado por factores externos tales como el nivel socioeconómico y cultural de las familias, el nivel socioeconómico del barrio de la escuela, el género del estudiante, su rendimiento previo, origen y lengua materna; de esta manera es posible determinar la influencia real de las variables relativas al porcentaje de tiempo que los docentes destinan a las diferentes tareas estudiadas. Así, en esta investigación trabajamos con tres tipos de variables: variables de rendimiento, o producto; variables de ajuste y variables sobre el porcentaje de tiempo que los docentes destinan a las diferentes tareas, o explicativas.

a) Variables de producto:

- Rendimiento en Matemáticas y Rendimiento en Lectura. Ambas estimadas mediante Teoría de Respuesta al Ítem (TRI) y escaladas con una media de 250 y una desviación típica de 50.

b) Variables contextuales de ajuste:

- Rendimiento previo en Lectura y en Matemáticas, obtenido a través de una prueba paralela a la de los rendimientos finales aplicada al comienzo del curso escolar. También estimadas mediante TRI y escaladas con una media de 250 y una desviación típica de 50.

- Nivel socioeconómico de la familia del estudiante, variable obtenida a partir de la profesión de los padres y de las posesiones familiares. Variable tipificada.

- Nivel cultural de la familia del estudiante, obtenida como promedio de la titulación máxima conseguida por ambos padres. Variable tipificada.

- Nivel socioeconómico del barrio donde se ubica la escuela, a partir de la opinión de la dirección de la escuela. Variable tipificada.

- Género del estudiante. Variable dummy.

- Origen del estudiante, nativo-inmigrante. Variable dummy.

- Lengua materna del estudiante, español u otra. Variable dummy.

- Género del docente. Variable dummy.

- Edad del docente, variable continua centrada en la moda. 
- Experiencia docente, años de experiencia que el docente tiene en su profesión. Variable continua.

- Antigüedad del docente, años que el docente lleva trabajando en la actual escuela. Variable continua.

c) Variables sobre el Porcentaje de Tiempo que los docentes destinan a las diferentes tareas, explicativas. Todas ellas se presentan como puntuaciones directas centradas en la media.

- Porcentaje de tiempo destinado a Planificar las clases.

- Porcentaje de tiempo destinado a Preparar evaluaciones.

- Porcentaje de tiempo destinado a Corregir ejercicios.

- Porcentaje de tiempo destinado a Tutorías con los estudiantes.

- Porcentaje de tiempo destinado a Reuniones con los padres.

- Porcentaje de tiempo destinado a Desarrollo profesional.

- Porcentaje de tiempo destinado a Tareas administrativas.

- Porcentaje de tiempo destinado a Trabajo en equipo con otros docentes.

- Porcentaje de tiempo destinado a Asistir a actividades extraescolares.

\section{Muestra}

La muestra estudiada está conformada por 5.610 estudiantes de 256 aulas de tercer curso de Primaria de 97 escuelas situadas en nueve países de Iberoamérica. La selección de dicha muestra se realizó de tal forma que, por una parte, se consiguiera una representatividad ecológica de los centros $\mathrm{y}$, por otra, se maximizara la varianza experimental. Así, los casos estudiados en cada uno de los niveles y el procedimiento para seleccionarlos fueron los siguientes.

En primer lugar, la selección de los nueve países fue determinada por ser los que formaban parte del Convenio Andrés Bello, quien financió el estudio: Bolivia, Chile, Colombia, Cuba, Ecuador, España, Panamá, Perú y Venezuela. En todo caso, la presencia de países de América del Sur, Centroamérica, el Caribe y Europa, junto con la diversidad en la situación social, económica y educativa de los nueve países implicados, permite afirmar que se aproximan a la realidad de la comunidad iberoamericana en su conjunto.

Como criterio general se planteó estudiar diez escuelas públicas de cada país en las que se impartiera Educación Básica/Primaria. Sin embargo, finalmente de Chile se analizó siete escuelas, de Venezuela nueve escuelas y de Ecuador 11 escuelas. Perú, por su parte, decidió ampliar el número de escuelas a 20 para tener un mayor número de datos para hacer sus propios cálculos. Análisis realizados previamente indican que esta ampliación de la muestra de Perú no altera los resultados. Con todo ello, se trabajó con 97 escuelas de 9 países (tabla 1). 
Tabla 1. Muestra del estudio: número de escuelas, docentes y estudiantes

\begin{tabular}{lccc}
\hline & EscuELAS & DoCENTES & EsTUDIANTES \\
\hline Bolivia & 10 & 30 & 662 \\
Chile & 7 & 18 & 409 \\
Colombia & 10 & 21 & 466 \\
Cuba & 10 & 38 & 694 \\
Ecuador & 11 & 26 & 662 \\
España & 10 & 21 & 328 \\
Panamá & 10 & 29 & 470 \\
Perú & 20 & 48 & 1565 \\
Venezuela & 9 & 25 & 354 \\
\hline Total & 97 & 256 & 5.610 \\
\hline
\end{tabular}

Para la selección de escuelas dentro de cada país se utilizaron dos criterios jerarquizados: en primer lugar, se eligieron cuatro escuelas cuyos estudiantes obtienen resultados muy por encima de los esperable teniendo en cuenta la situación socioeconómica y cultural de las familias, tres cuyos estudiantes tengan resultados mucho más bajos de lo esperable, y las tres restantes con estudiantes que obtienen resultados promedio para cada país. Dada la imposibilidad de tener, a priori, una estimación del rendimiento de las escuelas, se partió de los resultados de evaluaciones nacionales o internacionales, así como la opinión de expertos, tales como los inspectores. Para obtener una cierta representatividad ecológica, se utilizaron tres criterios: región geográfica, de tal forma que las escuelas reflejasen la variedad regional de cada país; hábitat de la localidad donde están ubicadas, para lo que seleccionaron escuelas situadas en una megaciudad (más de un millón de habitantes), zona urbana (entre un millón y 25.000 habitantes) y zona rural (menos de 25.000 habitantes); y tamaño de la escuelas, de tal forma que se analizaron escuelas grandes, medianas y pequeñas, en función del tamaño medio de las escuelas en cada país. El objetivo final que se buscó es que fueran representativas de la diversidad de las escuelas públicas del país. Respecto a las aulas, se decidió estudiar los grupos de tercer grado de
Educación Primaria/Básica (8/9 años de edad modal). De esta forma, se seleccionaron todas las aulas de tercer grado de las escuelas ya elegidas. En caso de que alguna de ellas tuviera más de cinco aulas de ese grado, se eligieron cinco al azar. En cada aula seleccionada se trabajó con los datos de todos los estudiantes, sus familias y sus docentes.

Se recogió información de 7.428 niños y niñas y 262 docentes. De ellos, generaron datos y fueron la muestra final los ya comentados 5.610 estudiantes de 256 aulas, situadas en 97 escuelas de los nueve países.

La muestra de alumnos tiene las siguientes características:

- El 54,8\% de ellos son niñas y el 45,2\%, niños (3.074 y 2.536, respectivamente).

- El 89,1\% de los estudiantes tenían 8 o 9 años. El 3,2\% menos de esa edad y el $7,7 \%$ restante más.

- El 92,7\% de los alumnos tiene el español como lengua materna y el 7,3\% otra, mayoritariamente lengua indígena (5.194 y 409 alumnos, respectivamente).

- El 95,9\% de los estudiantes son nativos del país donde estudian, y un 4,1\% son inmigrantes. 
La muestra de los docentes participantes en el estudio tiene las siguientes características:

- El 78\% son mujeres y el 22\% hombres.

- El 33\% tiene menos de 40 años de edad, el $43 \%$ tiene entre 40 y 50 años, y el $24 \%$ tiene más de 50 años.

- Según los años de experiencia como docente, el 54\% tiene hasta de 15 años de experiencia como docente, frente al $46 \%$ que tiene más de 16 años de experiencia.

- Según los años que lleva trabajando en la escuela, el 25\% lleva menos de 2 años en la escuela, otro $25 \%$ lleva hasta 5 años; el $28 \%$ entre 6 y 10 años, y un 22\% lleva trabajando en la escuela más de 10 años.

El análisis de la distribución del tiempo empleado por los docentes muestra que:
- La tarea a la que más tiempo se destina, un $17,2 \%$ (3h), es a Planificar las clases. Corregir los ejercicios de los estudiantes requiere de más de $2 \mathrm{~h}$ y media para los docentes, el $15 \%$ de su tiempo. Las siguientes tareas a las que se dedica más tiempo son el Desarrollo profesional y el Trabajo en equipo con otros docentes, $13,2 \%$ y 11,5\% respectivamente. En la parte baja del ranking de las tareas más desarrolladas, en términos de tiempo dedicado, se sitúan la realización de Tareas de carácter administrativo $(1,2 \mathrm{~h}$ a la semana) y es especialmente significativo que tener tutorías con sus estudiantes sea la tarea a la que de media los docentes dedican menos tiempo, poco más una hora (tabla 2).

Tabla 2. Distribución del tiempo semanal que los docentes dedican a las actividades no lectivas

\begin{tabular}{lccc}
\hline & MEDIA (EN HORAs) & DT & \% \\
\hline Planificar las clases & 3,0 & 1,5 & 17,2 \\
Preparar evaluaciones a los estudiantes & 2,1 & 1,3 & 12 \\
Corregir ejercicios de los estudiantes & 2,6 & 1,6 & 14,9 \\
Tutorías con los estudiantes & 1,1 & 1,1 & 6,3 \\
Reuniones con las familias & 1,4 & 1,0 & 8,0 \\
Desarrollo profesional & 2,3 & 1,8 & 13,2 \\
Tareas administrativas & 1,2 & 1,4 & 6,9 \\
Trabajo en equipo con otros docentes & 2,0 & 1,5 & 11,5 \\
Asistencia a actividades extracurriculares & 1,7 & 1,5 & 9,8 \\
\hline
\end{tabular}

\section{Instrumentos}

En esta investigación se utilizaron dos tipos de instrumentos:

a) Pruebas de Rendimiento en Matemáticas y las pruebas de Rendimiento en Lectura.

Se utilizaron las desarrolladas por el Laboratorio Latinoamericano de Evaluación de Calidad de la Educación para su primer estudio internacional (LLECE, 2001). Se trata de dos pruebas equivalentes para cada materia (llamadas pruebas A y B). Las pruebas se diseñaron y corrigieron a partir de la Teoría de Respuesta al Ítem (en concreto el modelo de un parámetro o modelo de Rasch). Las dos pruebas de Matemáticas están compuestas por 31 ítems de respuesta múltiple con cuatro alternativas cada uno. La fiabilidad de estas pruebas de Rendimiento en Matemáticas, medida a través del alpha de Cronbach, es de $\alpha$ $=0,89$ tanto para la prueba $\mathrm{A}$ (rendimiento previo) y para la prueba B (rendimiento final). 
Las dos pruebas de Rendimiento en Lectura, por su parte, están compuestas por 19 preguntas cerradas con cuatro posibles alternativas y una pregunta final abierta. Las pruebas de Rendimiento en Lectura tienen un índice de fiabilidad de $\alpha=0,92$ para la prueba A y de $\alpha=0,93$ para la prueba B.

b) Cuestionarios

En la investigación se elaboraron y utilizaron tres cuestionarios diferentes dirigidos a distintos colectivos escolares: uno para los docentes de las aulas seleccionadas (Cuestionario para los profesores del aula); otro para los estudiantes (Cuestionario general del estudiante), y un tercero para sus familias (Cuestionario para las familias).

El Cuestionario para los profesores del aula está compuesto por casi 195 cuestiones cerradas de todo tipo: desde preguntas tipo Likert, de respuesta graduada o de frecuencia, hasta cuestiones de respuesta numérica. Entre ellas, se plantean una batería de cuestiones relativas a la Distribución del Tiempo del Docente. Su fiabilidad, medida con el alpha de Cronbach es de $\alpha=0,85$.

El Cuestionario para los estudiantes se compone de 35 cuestiones sencillas en forma de preguntas cerradas, en general con cinco alternativas de respuesta, y obtiene información, entre otros elementos, de sus características personales. Este cuestionario tiene una fiabilidad de $\alpha=0,79$.

El Cuestionario para las familias, por último, está conformado por 41 cuestiones cerradas entre las que se encuentran preguntas sobre las posesiones familiares, la profesión del padre y de la madre o nivel de estudios máximos alcanzado por cada uno de ellos. Su fiabilidad es de $\alpha=0,84$.

Todos los instrumentos, tanto de rendimiento como los cuestionarios, fueron aplicados en español, por ser esta la lengua vehicular en todas las escuelas estudiadas.

\section{Trabajo de Campo}

En cada país, los datos fueron recogidos por un equipo de investigación especialista en la temática. El trabajo de campo se realizó en dos momentos diferenciados. En primer lugar, al comienzo del curso escolar se aplicó la Prueba de Matemáticas A y la Prueba de Lectura A, y los datos de ambas se consideraron como rendimiento previo. El resto de los instrumentos se aplicaron al finalizar el curso y por el mismo equipo que realizó la primera fase.

\section{Análisis de datos}

Como estrategia de análisis datos se utilizaron Modelos Multinivel de cuatro niveles de análisis (estudiante, aula, escuela y país). Su uso, habitual en estudios de este tipo (p.e. Creemers, Kyriakides \& Sammons, 2010; Goldstein, 2011; Martínez-Garrido \& Murillo, 2014), se justifica por trabajar con datos de diferentes niveles de análisis. Efectivamente, mientras que las variables dependientes y de ajuste son de estudiante, las variables explicativas son de nivel aula.

El proceso de modelado multinivel para cada una de las variables producto fue:

a) Estimar el modelo nulo (Modelo 1), sólo con la variable producto.

b) Calcular el modelo con las variables de ajuste (Modelo 2).

c) Incluir en el modelo ajustado las variables referidas a las Distribución del Tiempo del Docente de forma independiente (Modelos 3).

d) El último paso del proceso de modelaje consiste en la estimación del modelo final con la incorporación de todas las variables seleccionadas por su significatividad en el modelo teórico (Modelo 4).

De esta forma se estimaron dos modelos multinivel (uno para cada variable de producto) análogos al siguiente: 


$$
\begin{aligned}
& \mathrm{y}_{\mathrm{ijkl}}=\beta_{0 \mathrm{jkl}}+\beta_{1 \mathrm{l}} \mathrm{NSE} \_ \text {escuela } \mathrm{kl}_{1}+\beta_{2 \mathrm{jkl}} \mathrm{NCult}_{\mathrm{ijkl}}+\beta_{3 \mathrm{jkl}} \mathrm{NSE}_{\mathrm{ijkl}}+\beta_{4 \mathrm{jkl}} \text { Genero }_{\mathrm{ijkl}}+\beta_{5 \mathrm{jkl}} \mathrm{Rdto}_{-} \text {prev }_{\mathrm{ijkl}}+ \\
& \beta_{6 \mathrm{jkl}} \text { Origen }_{\mathrm{ijkl}}+\beta_{7 \mathrm{jkl} L e n g u a \_m a t e r n a} \mathrm{ijkl}_{1}+\beta_{8 \mathrm{kl}} \text { Genero_doc }{ }_{\mathrm{jkl}}+\beta_{9 \mathrm{kl}} \text { Edad_doc }_{\mathrm{jkl}}+ \\
& \beta_{10 \mathrm{kl}} \text { Experiencia_doc } \mathrm{jkl}+\beta_{11 \mathrm{kl}} \text { Antiguedad_doc } \mathrm{jkl}+\beta_{12 \mathrm{kl}} \text { VDistribución_Tiempo1 } 1_{\mathrm{jkl}}+\ldots \text {. } \\
& +\beta_{20 \mathrm{kl}} \text { VDistribución_Tiempo9 } 9_{\mathrm{jkl}}+\varepsilon_{\mathrm{ijkl}} \\
& \beta_{0 \mathrm{jkl}}=\beta_{0}+\varphi_{0 \mathrm{l}}+v_{0 \mathrm{kl}}+\mu_{0 \mathrm{jkl}} \\
& \beta_{11}=\beta_{1}+\varphi_{11} \\
& \beta_{2 j \mathrm{kl}}=\beta_{2}+\varphi_{2 \mathrm{l}}+v_{2 \mathrm{kl}}+\mu_{2 \mathrm{jkl}} \ldots \beta_{7 \mathrm{jkl}}=\beta_{7}+\varphi_{7 \mathrm{l}}+\nu_{7 \mathrm{kl}}+\mu_{7 \mathrm{jkl}} \\
& \beta_{8 \mathrm{kl}}=\beta_{8}+\varphi_{8 \mathrm{l}}+v_{8 \mathrm{kl}} \ldots \beta_{16 \mathrm{kl}}=\beta_{20}+\varphi_{20 \mathrm{l}}+v_{20 \mathrm{kl}}
\end{aligned}
$$

Con:

$$
\begin{aligned}
& {\left[\varepsilon_{0 \mathrm{ijkl}}\right] \sim \mathrm{N}\left(0, \Omega_{\varepsilon}\right): \Omega_{\varepsilon}=\left[\sigma^{2} \varepsilon_{0}\right]} \\
& {\left[\mu_{0 \mathrm{jk}}\right] \sim \mathrm{N}\left(0, \Omega_{\mu}\right): \Omega_{\mu}=\left[\sigma^{2} \mu_{0}\right]} \\
& {\left[v_{0 \mathrm{kl}}\right] \sim \mathrm{N}\left(0, \Omega_{v}\right): \Omega_{v}=\left[\sigma^{2} v_{0}\right]} \\
& {\left[\varphi_{01}\right] \sim \mathrm{N}\left(0, \Omega_{\varphi}\right): \Omega_{\varphi}=\left[\sigma^{2} \varphi_{0}\right]}
\end{aligned}
$$

\section{Donde:}

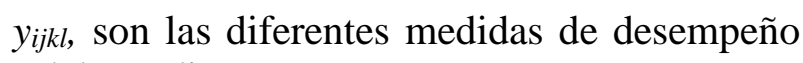
del estudiante,

NSE_escuela $a_{k l}$, es el nivel socio-económico del barrio donde está la escuela,

NCultijkl, es el nivel cultural de la familia del estudiante,

$N S E_{i j k l}$, es el nivel socio-económico de la familia del estudiante,

Genero $_{i j k l}$, si el estudiante es niño o niña,

Rend_previo ${ }_{i j k l}$, es el rendimiento previo tanto de Lectura como Matemáticas obtenido a comienzo del curso,

Origen $_{i j k l}$, es país de origen del estudiante: nativo o inmigrante,

Lengua_materna $a_{i j k l}$, si el estudiante habla una lengua diferente al castellano,

Género_doc $j k l$, si el docente es hombre o mujer,

Edad_docjkl, la edad del docente,

Experiencia_doc $j k l$, cantidad de años de experiencia que el docente tiene en su profesión,
Antigüedad_docjkl, número de años que el docente lleva trabajando en la actual escuela,

Distribución_Tiempojkl, se refiere a las nueve tareas estudiadas en las que el docente distribuye su tiempo. Estas variables son:

- Porcentaje de tiempo destinado a Planificar las clases,

- Porcentaje de tiempo destinado a Preparar evaluaciones a los estudiantes,

- Porcentaje de tiempo destinado a Corregir ejercicios,

- Porcentaje de tiempo destinado a Tutorías con los estudiantes,

- Porcentaje de tiempo destinado a Reuniones con las familias,

- Porcentaje de tiempo destinado a Desarrollo profesional,

- Porcentaje de tiempo destinado a Trabajo en equipo con otros docentes,

- Porcentaje de tiempo destinado a Tareas de tipo administrativo, $\mathrm{y}$

- Porcentaje de tiempo destinado a Asistencia a actividades extraescolares. 


\section{Resultados}

El objetivo de este estudio es determinar la incidencia del porcentaje de tiempo que los docentes iberoamericanos destinan a cada una de las tareas sobre el rendimiento académico de los estudiantes de Educación Primaria.
Con el fin de comprobar la relación entre las diferentes variables explicativas y ambas variables de producto, llevamos un análisis de correlación entre las variables implicadas (tabla 3). Como era esperable, todas las variables correlacionan con las dos medidas de rendimiento.

Tabla 3. Índices de correlación entre las variables explicativas y variables de producto

\begin{tabular}{|c|c|c|c|c|c|c|c|c|c|c|}
\hline & $\begin{array}{c}\text { RTO. } \\
\text { LECTURA }\end{array}$ & $\begin{array}{c}\text { RTO. } \\
\text { MATEMÁTICAS }\end{array}$ & $\mathbf{I}$ & II & III & IV & $\mathbf{V}$ & VI & VII & VIII \\
\hline I. Planificar las clases & ,256* & ,233* & & & & & & & & \\
\hline II. Preparar evaluaciones &, $224^{*}$ &, $188^{*}$ &, $559^{*}$ & & & & & & & \\
\hline III. Corregir ejercicios & $175^{*}$ &, $162^{*}$ & ,429* &, $466^{*}$ & & & & & & \\
\hline IV. Tutorías con los estudiantes &, $136^{*}$ &, $121^{*}$ & ,304* & ,354* & ,216* & & & & & \\
\hline V. Reuniones con las familias &, $11^{*}$ &, $028^{*}$ &, $115^{*}$ &, $088^{*}$ &, $113^{*}$ & ,253* & & & & \\
\hline VI. Desarrollo profesional &, $118^{*}$ &, $104^{*}$ &, $264^{*}$ &, $247^{*}$ &, $166^{*}$ &, $291^{*}$ & ,288* & & & \\
\hline VII. Tareas administrativas &,$- 073^{*}$ &,$- 106^{*}$ &, $071^{*}$ &, $154^{*}$ & ,116* &, $169^{*}$ & ,195* & ,379* & & \\
\hline $\begin{array}{l}\text { VIII. Trabajo en equipo } \\
\text { docente }\end{array}$ &, $060^{*}$ &, $063^{*}$ &, $220^{*}$ &, $224^{*}$ &, $170^{*}$ & ,263* &, $215^{*}$ & ,392* &, $280^{*}$ & \\
\hline IX. Asistencia a act. extracurr. &,$- 085^{*}$ &,$- 056^{*}$ & ,026 &, $047^{*}$ & ,016 &, $190^{*}$ & ,301* & ,385* & ,307 & ,306* \\
\hline
\end{tabular}

Nota: * significativo con $\alpha=0,01$.

Dado que las variables del profesorado (su género, su edad, los años de experiencia en la profesión como maestro y la antigüedad en la escuela) podrían condicionar la variable independiente (distribución del tiempo docente), analizamos la relación entre dichas variables. La tabla 4 muestra que las variables de docente son variables que están correlacionan con la distribución que el tiempo no lectivo que el docente lleve a cabo. Además, la prueba T-Student para grupos independientes indica que también el género del docente influye en cómo éste distribuya su tiempo no lectivo en la escuela. 
Martínez-Garrido, Cynthia \& Murillo, F. Javier (2016). Incidencia de la distribución del tiempo no lectivo de los docentes en Educación Primaria en el aprendizaje de sus estudiantes. RELIEVE, 22(2), art. 1. doi: http://dx.doi.org/10.7203/relieve.22.2.9433

Tabla 4. Relación entre las variables del profesorado y las variables explicativas

\begin{tabular}{lccccc}
\hline & \multicolumn{3}{c}{ CORRELACIÓN } & & T-STUDENT \\
\cline { 2 - 4 } \cline { 5 - 6 } & EDAD & EXPERIENCIA & ANTIGÜEDAD & & GÉNERO \\
\hline Planificar las clases &,$- 186^{* *}$ &,$- 077^{* *}$ &,$- 029^{*}$ & & $-9,367^{* *}$ \\
Preparar evaluaciones &,$- 055^{* *}$ &, 025 &, $092^{* *}$ & & $-15,338^{* *}$ \\
Corregir ejercicios &,$- 049^{* *}$ &, $030^{*}$ &, $070^{* *}$ & & $-6,886^{* *}$ \\
Tutorías con los estudiantes &,- 005 &, 002 &, $065^{* *}$ & & $-11,533^{* *}$ \\
Reuniones con las familias &, $037^{* *}$ &,$- 036^{* *}$ &, 009 & & $-4,049^{* *}$ \\
Desarrollo profesional &,$- 128^{* *}$ &,$- 105^{* *}$ &,$- 079^{* *}$ & & $-14,418^{* *}$ \\
Tareas administrativas &, $088^{* *}$ &, 025 &, $042^{* *}$ & & $-3,339^{* *}$ \\
Trabajo en equipo docente &,$- 036^{* *}$ &,$- 054^{* *}$ &, $034^{*}$ & & $-11,594^{* *}$ \\
Asistencia a actividades extracurr. &,$- 065^{* *}$ &,$- 102^{* *}$ &,- 024 & & $-12,35^{* *}$ \\
\hline
\end{tabular}

Notas:* significativo con $\alpha=0,05 ; * *$ significativo $\operatorname{con} \alpha=0,01$.

Para alcanzar el objetivo fijado se realizan dos modelamientos multinivel de cuatro niveles (estudiante, aula, escuela y país), uno para cada variable de producto: rendimiento en Lectura y rendimiento en Matemáticas, con cuatro grandes pasos.

En primer lugar, la estimación del Modelo nulo (Modelo 1), sólo con la variable producto. En segundo término, el Modelo con las variables de ajuste (Modelo 2). A continuación se introducen una a una cada variable explicativa en el Modelo 2, con lo que se obtienen nueve modelos, uno para cada variable (Modelos 3). Y, por último, el Modelo final (Modelo 4) que contiene tan sólo aquellas variables explicativas que hacen con una aportación significativa en el modelo anterior. Los modelos nulo, ajustado y con variables explicativas para cada una de las variables de producto aparecen en las tablas 5 y 6 .

Los resultados de los Modelos 2 (modelos ajustados, uno para cada variable de producto) ofrecen algunos interesantes datos que, aún no estando directamente relacionados con los objetivos de la investigación, es interesante comentarlos. Entre estos resultados "colaterales", pueden ser señalados los siguientes elementos:

- El nivel socioeconómico de la familia afecta al rendimiento académico de los estudiantes. Más concretamente los datos señalan que por cada desviación típica que aumenta el nivel socio-económico de los estudiantes, su rendimiento en Lectura aumenta 1,92 puntos y 2,67 puntos en Matemáticas (recordamos que estas variables estas medidas con una media de 250 y una desviación de 50).

- Por cada desviación típica que el nivel cultural de las familias aumenta, el rendimiento en Lectura aumenta 1,5 puntos y 3,5 puntos en Matemáticas.

- El impacto que el nivel socioeconómico del barrio donde se sitúa la escuela también cuenta con un importante efecto sobre el rendimiento de los estudiantes. Según nuestros resultados, aquellos estudiantes que acuden a escuelas situadas en contextos favorecidos obtienen mejores rendimientos. En concreto, por cada desviación típica que aumenta el nivel socioeconómico de la escuela, los estudiantes obtienen 6 puntos más, tanto en Lectura como en Matemáticas.

- El género está asociado al desempeño, pero de una forma diferente para cada área de conocimiento. Según nuestros resultados, los niños obtienen mejores puntuaciones en Matemáticas (3 puntos más) y las niñas en Lectura (3,5 puntos más).

- Ser inmigrante afecta de manera significativa a los resultados de los estudiantes en Matemáticas, en concreto, estos estudiantes obtienen 8 puntos menos. 
- Aquellos estudiantes que tengan una lengua materna diferente al castellano obtienen 4 puntos menos en su rendimiento en Lectura. La lengua materna parece no contar con un impacto significativo sobre el rendimiento de los estudiantes en Matemáticas.

- Como no podía ser de otra forma, el rendimiento previo impacta de manera significativa sobre el rendimiento final. Por cada punto de más que tenga un estudiante, subirá 0,41 y 0,39 puntos en Matemáticas y Lectura, respectivamente.

- Las variables relativas al docente, su género, edad, experiencia en la profesión docente y antigüedad en la escuela no realizan una aportación estadísticamente significativa en los modelos, por lo que no parecen influir en ninguno de los dos rendimientos.

Tabla 5. Resultados del proceso de modelamiento multinivel de cuatro niveles para Rendimiento en Lectura

\begin{tabular}{|c|c|c|c|c|c|c|}
\hline & \multicolumn{2}{|c|}{ MODELO 1} & \multicolumn{2}{|c|}{ MODELO 2} & \multicolumn{2}{|c|}{ MODELOS 3} \\
\hline & B & EE & B & EE & B & EE \\
\hline \multicolumn{7}{|l|}{ Parte fija } \\
\hline Intercepto & 276,64 & 7,80 & 176,77 & 4,83 & & \\
\hline NSE escuela & & & $6,08^{*}$ & 1,73 & & \\
\hline Nivel cultural familia & & & $1,49^{*}$ & 0,48 & & \\
\hline NSE familia & & & $1,94^{*}$ & 0,52 & & \\
\hline Género (varón/mujer) & & & $3,50^{*}$ & 0,81 & & \\
\hline Rendimiento Previo & & & $0,39^{*}$ & 0,01 & & \\
\hline Lengua materna & & & $-4,32^{* *}$ & 2,09 & & \\
\hline Nativo/Inmigrante & & & NS & & & \\
\hline Género docente (varón/mujer) & & & NS & & & \\
\hline Edad docente & & & NS & & & \\
\hline Experiencia docente & & & NS & & & \\
\hline Antigüedad en la escuela & & & NS & & & \\
\hline \% tiempo Planificar las clases & & & & & $2,37^{* *}$ & 1,08 \\
\hline$\%$ tiempo Preparar evaluaciones & & & & & $3,61^{*}$ & 1,09 \\
\hline$\%$ tiempo Corregir ejercicios & & & & & $1,78^{* *}$ & 0,88 \\
\hline \% tiempo Tutorías con los estudiantes & & & & & $1,21+$ & 0,89 \\
\hline \% tiempo Reuniones con la familia & & & & & $0,35+$ & 1,13 \\
\hline \% tiempo Desarrollo profesional & & & & & $0,24+$ & 0,69 \\
\hline \% tiempo Tareas administrativas & & & & & $-1,29 * * *$ & 0,77 \\
\hline$\%$ tiempo Trabajo en equipo docente & & & & & $1,67 * *$ & 0,84 \\
\hline \% tiempo Asistir a actividades extrac. & & & & & $-1,04+$ & 0,72 \\
\hline \multicolumn{7}{|l|}{ Parte aleatoria } \\
\hline Entre países & 510,08 & 257,90 & 126,86 & 66,10 & & \\
\hline Entre escuelas & 293,51 & 56,86 & 67,06 & 20,594 & & \\
\hline Entre aulas & 118,50 & 19,03 & 108,66 & 16,645 & & \\
\hline Entre estudiantes & 1026,65 & 19,83 & 830,15 & 16,03 & & \\
\hline
\end{tabular}

Notas: * significativo con $\alpha=0,01$; * significativo con $\alpha=0,05$; ** significativo con $\alpha=0,1$; NS: no significativo; + : no significativo.

Fuente: Elaboración propia. 
Tabla 6. Resultados del proceso de modelamiento multinivel de cuatro niveles para Rendimiento en Matemáticas

\begin{tabular}{|c|c|c|c|c|c|c|}
\hline & \multicolumn{2}{|c|}{ MODELO 1} & \multicolumn{2}{|c|}{ MODELO 2} & \multicolumn{2}{|c|}{ MODELOS 3} \\
\hline & B & EE & B & EE & B & EE \\
\hline \multicolumn{7}{|l|}{ Parte fija } \\
\hline Intercepto & 266,45 & 9,21 & 164,24 & 6,49 & & \\
\hline NSE escuela & & & $6,41^{*}$ & 2,42 & & \\
\hline Nivel cultural familia & & & $3,50^{*}$ & 0,54 & & \\
\hline NSE familia & & & $2,67^{*}$ & 0,59 & & \\
\hline Género (varón/mujer) & & & $-3,09^{*}$ & 0,92 & & \\
\hline Rendimiento Previo & & & $0,41^{*}$ & 0,01 & & \\
\hline Lengua materna & & & NS & & & \\
\hline Nativo/Inmigrante & & & $-8,27^{* *}$ & 3,65 & & \\
\hline Género docente (varón/mujer) & & & NS & & & \\
\hline Edad docente & & & NS & & & \\
\hline Experiencia docente & & & NS & & & \\
\hline Antigüedad & & & NS & & & \\
\hline \% tiempo Planificar las clases & & & & & $2,73^{* * *}$ & 1,60 \\
\hline \% tiempo Preparar evaluaciones & & & & & $3,17^{* *}$ & 1,62 \\
\hline \% tiempo Corregir ejercicios & & & & & $1,42^{+}$ & 1,30 \\
\hline \% tiempo Tutorías con los estudiar & & & & & $-0,45^{+}$ & 1,34 \\
\hline \% tiempo Reuniones con la familia & & & & & $2,85^{* * *}$ & 1,68 \\
\hline \% tiempo Desarrollo profesional & & & & & $0,05^{+}$ & 1,03 \\
\hline \% tiempo Tareas administrativas & & & & & $-0,94^{+}$ & 1,11 \\
\hline \% tiempo Trabajo en equipo docen & & & & & $0,58^{+}$ & 1,23 \\
\hline \% tiempo Asistir a actividades extr & & & & & $-0,59^{+}$ & 1,03 \\
\hline
\end{tabular}

\section{Parte aleatoria}

\begin{tabular}{lcccc} 
Entre países & 709,18 & 360,65 & 242,85 & 126,46 \\
Entre escuelas & 395,76 & 85,04 & 110,21 & 41,23 \\
Entre aulas & 283,23 & 38,85 & 306,13 & 39,82 \\
Entre estudiantes & 1261,93 & 24,39 & 1064,16 & 20,56 \\
\hline
\end{tabular}

Notas: * significativo con $\alpha=0,01 ; * *$ significativo con $\alpha=0,05 ; * * *$ significativo con $\alpha=0,1$; NS: no significativo; +: no significativo.

Partiendo del Modelo 2, para cada una de las dos variables producto, construimos nueve Modelos 3, uno por cada variable de Distribución de Tiempo considerada. Los resultados señalan que de las nueve tareas estudiadas a las que los docentes destinan su tiempo, seis tienen un coeficiente significativo en el modelo; es decir, impactan sobre el rendimiento de los estudiantes: Planificar las clases, Preparar las evaluaciones de los estudiantes, Corregir los ejercicios, Reunirse con las familias, Trabajar en equipo con el profesorado y Realizar tareas administrativas. Veamos cuál es su impacto descontando el impacto de las variables de ajuste:

- El porcentaje de tiempo que el docente destina a planificar sus clases impacta 
sobre el rendimiento académico de los estudiantes, más en Matemáticas que en Lectura. Por cada punto que el porcentaje de tiempo destinado a planificar sus clases aumenta (o disminuya), el rendimiento de los estudiantes aumenta (o disminuye) 2,7 puntos en Matemáticas y 2,3 puntos en Lectura. Recordemos que el rendimiento en Lectura y Matemáticas está medido con una media de 250 y desviación típica de 50 .

- La variable porcentaje de tiempo destinado a preparar las evaluaciones de los estudiantes hace una aportación significativa tanto para el modelo ajustado de Rendimiento en Lectura como para el de Rendimiento en Matemáticas. Los resultados encontrados muestran que esta aportación es mayor en Lectura que en Matemáticas. Así, por cada punto que aumenta el porcentaje de tiempo destinado a preparar evaluaciones, el rendimiento en Lectura aumenta 3,6 puntos, frente a los 3,1 puntos que mejora el rendimiento en Matemáticas. Recordemos que las variables de Distribución de Tiempo son puntuaciones directas centradas en la media.

- Por cada punto que aumenta el porcentaje de tiempo destinado a corregir los ejercicios de los estudiantes, su rendimiento en Lectura aumenta 1,8 puntos. Según los resultados encontrados, esta variable no hace una aportación significativa al Modelo 3 para la variable de producto rendimiento en Matemáticas.

- La variable porcentaje de tiempo destinado a reunirse con las familias de los estudiantes hace una aportación significativa al modelo ajustado de la variable rendimiento en Matemáticas. Por cada punto que este porcentaje de tiempo aumente, el rendimiento en Matemáticas se incrementa 2,8 puntos.

- Por cada punto que aumenta el tiempo destinado a trabajar en equipo, el rendimiento de los estudiantes en Lectura aumenta 1,6 puntos.

-El impacto generado sobre el modelo ajustado para rendimiento en Lectura de la variable porcentaje de tiempo destinado a realizar tareas administrativas es negativo. De esta forma, por cada punto porcentual que éste tiempo aumenta, el rendimiento de los estudiantes en Lectura baja 1,3 puntos.

- Por último, según nuestros resultados, las variables porcentaje de tiempo destinado a tutorías con los estudiantes, porcentaje de tiempo destinado a desarrollo profesional y porcentaje de tiempo destinado a asistir a actividades extraescolares no hacen un impacto significativo en el modelo ajustado por lo que no están asociadas al aprendizaje de los estudiantes.

El siguiente y último paso en la construcción del Modelo 4, o modelo final, para cada una de las variables de producto (tabla 7). Para llevarlo a cabo, se introducen conjuntamente tan sólo aquellas variables que han resultado significativas en la fase anterior del proceso de modelado. 
Tabla 7. Resultados del Modelo final para Rendimiento en Lectura y Rendimiento en Matemáticas

\begin{tabular}{lcccc}
\hline & \multicolumn{2}{c}{$\begin{array}{c}\text { RENDIMIENTO EN } \\
\text { LECTURA }\end{array}$} & \multicolumn{2}{c}{$\begin{array}{c}\text { RENDIMIENTO EN } \\
\text { MATEMÁTICAS }\end{array}$} \\
\cline { 2 - 5 } & B & EE & B & EE \\
\hline Parte fija & & & & \\
Intercepto & 176,86 & 4,70 & 163,45 & 6,46 \\
NSE escuela & $4,79^{*}$ & 1,68 & $5,41^{*}$ & 2,45 \\
Nivel cultural familia & $1,50^{*}$ & 0,48 & $3,49^{*}$ & 0,54 \\
NSE familia & $1,96^{*}$ & 0,52 & $2,65^{*}$ & 0,59 \\
Género (varón/mujer) & $3,50^{*}$ & 0,81 & $-3,10^{*}$ & 0,92 \\
Rendimiento Previo & $0,39^{*}$ & 0,01 & $0,41^{*}$ & 0,01 \\
Lengua materna & $-4,08^{* *}$ & 2,08 & - & \\
Nativo/Inmigrante & - & & $-8,22^{* *}$ & 3,65 \\
\hline \% tiempo Planificar las clases & $2,11^{* *}$ & 1,07 & $2,45^{* *}$ & 1,23 \\
\% tiempo Preparar evaluaciones & $3,32^{* *}$ & 1,33 & $2,91^{* *}$ & 1,45 \\
\% tiempo Reuniones con la familia & - & & $2,38^{* * *}$ & 1,20 \\
\% tiempo Tareas administrativas & $-1,25^{* *}$ & 0,63 & - & \\
\hline Parte aleatoria & & & & \\
Entre países & 119,29 & 61,85 & 240,19 & 124,85 \\
Entre escuelas & 56,28 & 18,49 & 112,61 & 40,69 \\
Entre aulas & 102,92 & 15,97 & 289,07 & 37,98 \\
Entre estudiantes & 830,15 & 16,03 & 1064,74 & 20,57 \\
\hline
\end{tabular}

Notas: * significativo con $\alpha=0,01 ; * *$ significativo con $\alpha=0,05 ; * * *$ significativo con $\alpha=0,1 ;-$ : indica que la variable no ha sido incluida en el modelo dado que su aportación individual es no significativa. Las variables: Género del docente, Edad del docente, Experiencia docente, Antigüedad en la escuela, \% tiempo Corregir ejercicios, \% tiempo Tutorías con los estudiantes, \% tiempo Trabajo en equipo docente, \% tiempo Desarrollo profesional, \% tiempo Asistir a actividades extracurriculares no aparecen en la tabla dado que su aportación fue no significativa.

\section{Discusión y Conclusiones}

El estudio realizado señala con claridad que cuanto mayor sea la dedicación del docente a tareas como planificar las clases, preparar las evaluaciones y reuniones con familias mayor será el rendimiento de los estudiantes en Lectura y Matemáticas. Por el contrario, los resultados obtenidos indican que aquellos docentes que más tiempo dedican a tareas administrativas hacen que sus estudiantes aprendan menos, concretamente, en Lectura.

Estos resultados son coherentes con los encontrados por Gran, Hindman y Stronge
(2010), Nonis, Philhours y Hudson (2006), o Walberg, y Paik (2000), entre otros. Así, por ejemplo, en el estudio desarrollado por Nye, Konstantopoulos y Hedges (2004) se señala que una mayor planificación de las clases genera un aumento de hasta 0,5 desviaciones típicas en el rendimiento de los estudiantes. O el trabajo de Gran, Hindman y Stronge (2010), que señala que el 52\% del éxito (o fracaso) logrado por los estudiantes en Matemáticas depende directamente de la planificación de la asignatura ideada por el docente.

También está ampliamente documentada por la investigación la importancia de la 
comunicación docente-familia para el aprendizaje de los estudiantes (p.ej., Castro, et al., 2015; De Carvalho, 2014; Niia et al.,;2015). Los resultados de estos recientes estudios confirman que es "imprescindible" que las familias conozcan la situación educativa de sus hijos y colaboren en su educación. Nuestros resultados no sólo confirman este hecho, sino que además muestran cómo impacta el tiempo que los docentes destinan a reunirse con las familias sobre el rendimiento de los estudiantes. Así, los datos obtenidos señalan que los docentes destinan el 8\% de su tiempo a reunirse con las familias y que por cada punto porcentual que éste tiempo se incremente, el rendimiento de los estudiantes en Matemáticas aumenta casi 3 puntos. Estos resultados son coherentes con los encontrados en el meta-análisis de Castro y otros (2015), al señalar que una mayor relación familia-escuela, mejora el rendimiento de los estudiantes.

Así mismo, según nuestros resultados, los docentes destinan una media de 1,2 horas a la semana a la realización de tareas administrativas, resultados coherentes con los aportados por Moya (2001) quién señaló que las tareas administrativas recibían el 0,7\% del tiempo de los docentes. Nuestros resultados apuntan un importante hecho, la dedicación del docente a tareas administrativas resulta contraproducente para con su principal labor en la escuela: conseguir que los estudiantes aprendan. Los datos analizados de 5.610 estudiantes de 9 países iberoamericanos confirman que por cada punto porcentual que aumenta el tiempo que los docentes destinan a realizar tareas administrativas el rendimiento de los estudiantes desciende 1,3 puntos en Lectura. Resultados que son coherentes con los aportados por DeStefano y Miksic (2007), quienes señalan que las escuelas donde más tareas administrativas se desempeñan ofrecen un $26 \%$ menos de oportunidades a sus estudiantes para aprender.

Quizá una de las mayores críticas que se le ha hecho a los estudios de eficacia es que utilizan un grupo muy restringido de variables de ajuste, dentro de las cuales no incluyen variables de proceso referidas a la vida social y cultural de las aulas y las escuelas (Scheerens, 1999; Martinic \& Pardo, 2003). Aunque pueda suponerse como una limitación, en este estudio hemos incluido un total de once variables de ajuste que responden a las características de los estudiantes, las familias, los docentes y la escuela.

Los resultados obtenidos tienen una inmediata aplicación práctica, tanto para la administración educativa y la dirección de los centros, como para los propios docentes.

Para la administración y la dirección de los centros, hay que recordar que si se quiere que los docentes hagan mejor si trabajo es necesario apoyarles, facilitarles las adecuadas condiciones laborales para ello. La tarea del docente no es sólo dar clase. Y se ha visto que el mayor o menor dedicación a esas tiempo mejora el rendimiento de los estudiantes porque hace que el docente haga un trabajo de mejor calidad. Es necesario que entre el tiempo remunerado de los docentes se encuentre el tiempo para preparar clases y corregir evaluaciones. Y también tiempo para atender y colaborar con las familias. Y ello significa no solo tiempo, también lugares donde el docente pueda trabajar concentrado y donde puede reunirse con padres y madres. Limitar en la medida de lo posible las tareas burocráticas que el docente debe hacer también hace que mejore su trabajo, seguramente porque su disminución le posibilita hacer "lo importante".

Es necesario que los docentes tengan presente que el tiempo dedicado a la preparación de las clases y de las evaluaciones es el tiempo mejor invertido. No importa si es la primera vez que imparten esa materia o llevan 20 años haciéndolo: los datos son claros, cuanto más tiempo, mejor. También hay que insistir en la importancia de dedicar tiempo a las familias. Solo con la colaboración docente-familia es posible conseguir el máximo desarrollo de los niños y niñas.

Futuras líneas de trabajo emergen a partir de los hallazgos del estudio. En primer lugar, es 
preciso seguir indagando cómo evoluciona la distribución del tiempo que hacen los docentes sobre determinadas tareas vista la influencia significativa que algunas de ellas tienen sobre el rendimiento académico de los estudiantes. Es relevante el tiempo que dediquen a ellas, pero también el tiempo que restan de dedicación a otras actividades. Cómo se organicen las estrategias pedagógicas en torno a las diferentes tareas a realizar por los docentes pauta la misión de la escuela, cuestión crucial al trabajar con países de una región tan inequitativa como es Iberoamérica. Una segunda línea ha de ser la Educación Secundaria en Iberoamérica; de manera que se identifiquen en esta etapa educativa la distribución temporal que hacen los docentes sobre estas tareas y los efectos que dicha distribución temporal generan sobre el rendimiento académico de los jóvenes estudiantes.

Estamos todos de acuerdo en considerar a los docentes como el elemento clave que determina la calidad del sistema educativo. Sin embargo, para que los y las docentes den lo mejor de sí hay que apoyarles. Demasiadas evaluaciones controlan sus conocimientos y su desempeño, demasiadas pocas verifican que su trabajo se desarrolla en unas condiciones laborales adecuadas, con tiempo suficiente para planificar clases y reunirse con los padres, con espacios y condiciones materiales adecuadas, con un salario digno para poder centrarse en exclusividad en su trabajo como docente....

El docente es el elemento clave del sistema educativo, $\mathrm{y}$ el resto debe confiar en ellos y apoyarles.

\section{Referencias}

Castro, M., Expósito-Casas, E., López-Martín, E., Lizasoain, L., Navarro-Asencio, E. \& Gaviria, J. L. (2015). Parental involvement on student academic achievement: A metaanalysis. Educational Research Review, 14, 33-46.

doi: http://dx.doi.org/10.1016/j.edurev.2015.01.002
Concha, C. (1996). Estudio de 32 escuelas de alta vulnerabilidad socioeconómica y altos resultados de aprendizaje. Santiago de Chile: Ministerio de Educación.

Creemers, B., Kyriakides, L., \& Sammons, P. (2010). Methodological advances in educational effectiveness research. Nueva York: Routledge.

De Carvalho, M. E. (2014). Rethinking familyschool relations: A critique of parental involvement in schooling. Nueva York: Routledge.

DeStefano, J. \& Miksic, E. (2007). School Effectiveness in Maissade, Haiti. Educational Quality Improvement Program. Washington, DC: Academy for Educational Development.

Eurydice (2013). Key data on teachers and school leaders in Europe. Luxemburgo: Publications Office of the European Union.

Gajardo, M. (2002). El tiempo y el aprendizaje en América Latina. Formas \& Reformas de la Educación-Series Políticas, 4(11), 6-15.

Gibson, S. (1984). Teacher efficacy: a construct validation. Journal of Educational Psychology, 76(4), 569-582. doi: http://dx.doi.org/10.1037/0022$\underline{0663.76 .4 .569}$

Goldstein, H. (2011). Multilevel Statistical Models. Londres: Wiley

Gran, L. W., Hindman, J. y Stronge, J. H. (2010). Planning, Instruction, and Assessment: Effective Teaching Practices. Nueva York: Eye on Education.

Hall, K. y Harding, A. (2003). A systematic review of effective literacy teaching in the 4 to 14 age range of mainstream schooling. Londres: Social Science Research Unit, Institute of Education.

Hiebert, J., Morris, A. K. \& Spitzer, S. M. (2009). Mathematical knowledge for teaching in planning and evaluating instruction: What can preservice teachers learn? Journal for Research in Mathematics Education, 40(5), 491-529. doi: http://dx.doi.org/10.1016/s0742$\underline{051 x(98) 00038-9}$ 
Husman, J., Duggan, M. A. \& Fishman, E. (2014). The teacher time bubble. En P. Richardson, S. Karabenick y H. Watt (Eds.), Teacher motivation: theory and practice (pp. 182-198). Nueva York: Routledge.

Kyriacou, C. (1987). Teacher stress and burnout: An international review. Educational Research, 29(2), 146-152. doi: http://dx.doi.org/10.1080/0013188870290207

Lavy, V. (2011). What makes an effective teacher? Quasi-experimental evidence. Cambridge, MA: National Bureau of Economic Research.

LLECE (2010). Factores asociados al logro cognitivo de los estudiantes de América Latina y el Caribe. Santiago de Chile: OREALC/UNESCO

Martínez-Garrido, C. (2015). Investigación sobre Enseñanza Eficaz. Un estudio multinivel para Iberoamérica. Tesis Doctoral. Universidad Autónoma de Madrid. España.

Martínez-Garrido, C. \& Murillo, F. J. (2014). Programas para la realización de Modelos Multinivel. Un análisis comparativo entre MLwiN, HLM, SPSS y Stata. REMA. Revista Electrónica de Metodología Aplicada, 12(2), $1-24$.

Martinic, S. \& Pardo, M. (2003). Aportes de la investigación educativa iberoamericana para el análisis de la Eficacia Escolar. En F. J. Murillo (Coord.), La investigación sobre eficacia escolar en Iberoamérica (pp. 93126). Bogotá: CIDE

Miñano, P. \& Castejón, J. L. (2011). Variables cognitivas y motivacionales en el rendimiento académico en Lengua y Matemáticas: un modelo estructural. Revista de Psicodidáctica 16(2), 203-230.

Montecinos, C. (2003). Desarrollo profesional docente y aprendizaje colectivo. Psicoperspectivas. Individuo y Sociedad, 2(1), 105-128.

Murillo, F. J. (Coord.) (2007). Investigación Iberoamericana sobre Eficacia Escolar. Bogotá: Convenio Andrés Bello.
Murillo, F. J. \& Hernández-Castilla, R. (2011). Factores escolares asociados al desarrollo socio-afectivo en Iberoamérica. RELIEVE, 17(2), art 2. doi: http://dx.doi.org/10.7203/relieve.17.2.4007

Murillo, F. J. \& Román, M. (2013). Docentes de educación primaria en América Latina con más de una actividad laboral: situación e implicaciones. Revista Mexicana de Investigación Educativa, 18(58), 893-924.

Murillo, F. J., Martínez-Garrido, C. \& Hernández-Castilla, R. (2011). Decálogo para una enseñanza eficaz. REICE. Revista Iberoamericana sobre Calidad, Eficacia y Cambio en Educación, 9(1), 6-27.

Niia, A., Almqvist, L., Brunnberg, E. \& Granlund, M. (2015). Student participation and parental involvement in relation to academic achievement. Scandinavian Journal of Educational Research, 59(3), 297315. doi: http://dx.doi.org/10.1080/00313831.2014.904 $\underline{421}$

Nonis, S. A., Philhours, M. \& Hudson, G. I. (2006). Where does the time do? A diary approach to business and marketing students' time use. Journal of Marketing Education, 28, 121-134. doi: http://dx.doi.org/10.1177/0273475306288400

Nye, B., Konstantopoulos, S. \& Hedges, L. V. (2004). How large are teacher effects? Educational Evaluation and Policy Analysis, 26(3), 237-257. doi: http://dx.doi.org/10.3102/01623737026003237

OCDE (2013). Informe TALIS 2013: estudio internacional sobre enseñanza-aprendizaje. París: OCDE.

Pennings, H., van Tartwijk, J., Wubbels, T., Claessens, L., van der Want, A. \& Brekelmans, M. (2014). Real-time teacherstudent interactions: A dynamic systems approach. Teaching and Teacher Education, 37(2), 183-193. doi: http://dx.doi.org/10.1016/j.tate.2013.07.016

Scheerens, J. (1999). Recent developments in school effectiveness research in the Netherlands. En T. Townsend, P. Clarke \& 
M. Ainscow (Eds.), Third Millenium Schools; a world of difference in effectiveness and improvement (pp. 143-159). Lisse: Swets \& Zeitlinger.

Shidler, L. (2009). The impact of time spent coaching for teacher efficacy on student achievement. Early Childhood Education Journal, 36(5), 453-460. doi: http://dx.doi.org/10.1007/s10643-008-0298-4

Vandenberghe, R. y Huberman, A.M. (Eds.). (1999). Understanding and preventing teacher burnout: A sourcebook of international research and practice. Cambridge: Cambridge University Press.

Vannest, K., Hagan-Burke, S. \& Parker, R. (2006). Teacher allocation and use of teacher time. College Statio, TX: Texas A\&M University.

Walberg, J. H. \& Paik, D. J. (2000). Effective educational practices. Ginebra: IBE/UNESCO.

\section{Autores / Authors}

To know more /

Saber más

Cynthia Martínez-Garrido (cynthialme@gmail.com).

Profesora en la Universidad de Zaragoza. Doctora en Educación por la Universidad Autónoma de Madrid (FPI-UAM). Editora de varias revistas de investigación científica: REICE. Revista Iberoamericana sobre Calidad, Eficacia y Cambio en Educación; Revista Internacional de Educación para la Justicia Social; Revista Latinoamericana de Educación Inclusiva. Página web de docencia: www.cmartinezgarrido.es. Su dirección postal es: Universidad de Zaragoza. Facultad de Ciencias Sociales y Humanas. Despacho nº 13. 44003-Teruel (España).

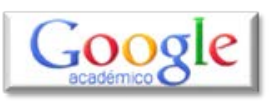

F. Javier Murillo (javier.murillo@uam.es).

Profesor titular del Área de Métodos de Investigación y Diagnóstico en Educación de la Universidad Autónoma de Madrid. Fue Coordinador General del Laboratorio Latinoamericano de Evaluación de la Calidad de la Educación (LLECE), de la UNESCO, y Director de Estudios del Centro de Investigación y Documentación Educativa (CIDE) del Ministerio de Educación de España. Es Coordinador de la Red Iberoamericana de Investigación sobre Cambio y Eficacia Escolar (RINACE), Director de la Revista Iberoamericana sobre Calidad, Eficacia y Cambio en Educación (REICE) y co-director de la Revista Iberoamericana de Evaluación Educativa. Ha trabajado como consultor en Investigación y Evaluación Educativas en diferentes países de América Latina y con distintas agencias internacionales UNESCO, OCDE y Convenio Andrés Bello-. Dirección postal: Facultad de

\section{RELIEVE}

\section{Revista ELectrónica de Investigación y $\mathbf{E V}$ aluación Educativa E-Journal of Educational Research, Assessment and Evaluation [ISSN: 1134-4032]}

(C) Copyright, RELIEVE. Reproduction and distribution of this articles it is authorized if the content is no modified and their origin is indicated (RELIEVE Journal, volume, number and electronic address of the document).

(C) Copyright, RELIEVE. Se autoriza la reproducción y distribución de este artículo siempre que no se modifique el contenido y se indique su origen (RELIEVE, volumen, número y dirección electrónica del documento). 